Meta

Journal des traducteurs

Translators' Journal

\title{
Figement et traduction : problématique générale
}

\section{Salah Mejri}

Volume 53, numéro 2, juin 2008

La traduction des séquences figées

The Translation of Frozen Sequences

URI : https://id.erudit.org/iderudit/018517ar

DOI : https://doi.org/10.7202/018517ar

Aller au sommaire du numéro

Éditeur(s)

Les Presses de l'Université de Montréal

ISSN

0026-0452 (imprimé)

1492-1421 (numérique)

Découvrir la revue

Citer cet article

Mejri, S. (2008). Figement et traduction : problématique générale. Meta, 53(2), 244-252. https://doi.org/10.7202/018517ar

\section{Résumé de l'article}

Après avoir montré l'importance du figement dans le fonctionnement de la langue et l'impact qu'il peut avoir sur les phénomènes de conceptualisation par la langue, il s'agit de voir comment on ne peut pas passer sous silence la trace de cette empreinte linguistique bien présente dans les séquences figées lors de la traduction. C'est seulement en admettant l'idée que la traduction est une négociation permanente entre forme et contenu qu'on pourrait obtenir un bon résultat. d'utilisation que vous pouvez consulter en ligne.

https://apropos.erudit.org/fr/usagers/politique-dutilisation/ 


\title{
Figement et traduction : problématique générale
}

\author{
SALAH MEJRI \\ Université Paris 13, Paris, France \\ salah.mejri@lli.univ-paris13.fr
}

\begin{abstract}
RÉSUMÉ
Après avoir montré l'importance du figement dans le fonctionnement de la langue et l'impact qu'il peut avoir sur les phénomènes de conceptualisation par la langue, il s'agit de voir comment on ne peut pas passer sous silence la trace de cette empreinte linguistique bien présente dans les séquences figées lors de la traduction. C'est seulement en admettant l'idée que la traduction est une négociation permanente entre forme et contenu qu'on pourrait obtenir un bon résultat.
\end{abstract}

\begin{abstract}
After having pointed out the importance of frozen structures in the functioning of language and its impact on the conceptualization phenomena through language, we intend to prove that this linguistic evidence cannot be ignored when translating frozen structures. It is only by admitting the fact that translating means a continuous transfer between form and content that a good result can be obtained.
\end{abstract}

\section{MOTS-CLÉS/KEYWORDS}

figement, traduction, déficit, séquences figées

\section{Figement et traduction}

Nous commençons par rappeler les éléments définitoires des deux termes de cette opposition en vue de préciser la perspective dans laquelle nous les rapprochons. Le figement, qui est un processus linguistique, engage toutes les dimensions du système. Puisqu'il s'agit d'un phénomène qui est à l'origine de la fixation dans le lexique d'unités syntagmatiques bien formées, il fournit des données précises sur les ingrédients linguistiques qui ont servi à la formation de telles unités, c'est-à-dire les règles en usage lors de la synchronie qui a vu naître le figement des séquences concernées. Cela est moins visible quand il s'agit de séquences figées (SF) actuelles, leur formation étant identique à celle des séquences libres ayant la même configuration; mais quand les séquences remontent à des synchronies antérieures comportant des spécificités non partagées ${ }^{1}$, la reconnaissance est immédiate. Cela se traduit par une fixation des mécanismes linguistiques en fonctionnement sur le plan à la fois phonologique, intonatif, rythmique, syntaxique, lexical et sémantique. Or puisqu'il s'agit d'unités initialement discursives, on est en droit de supposer qu'elles portent en elles les traces d'une conceptualisation individuelle et spontanée, qui témoigne d'usages linguistiques nécessairement représentatifs du linguistique comme du conceptuel. Nous présentons dans ce qui suit les principales caractéristiques du figement:

- $\quad$ il concerne toute la langue. Même s'il trouve sa pleine expression dans le lexique (les expressions idiomatiques, les formules, les proverbes, etc.), il ne s'y limite pas. Les travaux récents insistent sur la continuité entre ce qui est libre et ce qui est figé (Mejri 2000d; G. Gross 1996; etc.); 
- $\quad$ il crée ainsi une rupture qui s'inscrit dans une sorte de continuité avec les combinaisons libres; ce qui témoigne de l'extrême complexité du traitement du figement par le locuteur, qui est censé bien maîtriser le code, et le linguiste qui doit tenir compte de tous les paramètres qu'il implique;

- il véhicule ce qu'il y a de plus intrinsèque au système, c'est-à-dire l'idiomaticité en tant que forme de la substance de l'expression et du contenu ${ }^{2}$;

- la systémicité s'explique par son intervention en tant que pendant de la synonymie dans le système sémiotique qu'est la langue ${ }^{3}$;

- avec la polysémie, il est le siège de toutes les croyances partagées par la communauté linguistique; ce qui signifie que les SF, par leur structuration sémantique, trahissent la manière dont le monde est catégorisé par le langage;

- la signification des SF s'inscrit dans un continuum qui va de la transparence la plus totale (avoir froid, rendre justice, etc.) à l'opacité complète (manger les pissenlits par la racine, battre la campagne, etc.), en passant par une transparence plus ou moins altérée ou une opacité quelque peu aérée (vin gris, panier de crabes, etc.).

Quant à la traduction, nous l'appréhendons comme une activité qui consiste à transférer des contenus d'une langue à une autre. Pour nous, cette activité est irréductible à la déverbalisation, couramment invoquée pour en rendre compte. Cette vision des choses fait un raccourci méthodologique fondé sur une conception de la langue un peu particulière, qui dissocie expression et contenu. En d'autres termes, l'expression serait un simple habillage du contenu, dont elle peut être dissociée sans que cela porte le moindre préjudice au contenu "exprimé». Cette vision «modulaire», qui voit dans l'expression et le contenu deux modules autonomes, passe sous silence l'extrême complexité des relations qu'entretiennent ces deux niveaux du système linguistique. Certes certains concepts sont concevables indépendamment des codes dans lesquels ils sont exprimés, qu'ils soient linguistiques ou autres, surtout quand il s'agit de conceptualisation instantanée et individuelle, donc discursive, mais ce serait faire de la langue un simple moule modelable et modifiable à merci, d'une neutralité telle qu'il serait aisé et facile de passer de l'une à l'autre sans la moindre altération des contenus signifiés. Ce serait méconnaître l'importance de l'empreinte du système sur le contenu et ne pas tenir compte de facteurs intrinsèques et extrinsèques aux codes qui fondent l'idiomaticité de chaque code. Qu'on songe un peu à la grammaticalisation de certaines catégories, à la lexicalisation des significations et au figement des séquences syntagmatiques! Que faire dans ce cas de ces spécificités internes? Il en est de même des phénomènes externes qui agissent fortement sur la manière dont les langues codent le réel. Nous pensons en particulier au poids de la norme, à la fonction identitaire de la langue et à l'impact que les locuteurs ont sur cet outil d'expression. Ne pas tenir compte de telles dimensions, c'est tout simplement considérer la langue comme un système non inférentiel. Or parler, c'est inférer selon la structuration imposée par la langue.

Pour nous, traduire revient à «surfer» constamment et «dangereusement» sur deux systèmes en vue de transférer le maximum de contenu d'un code à un autre sans avoir la moindre illusion que cette opération n'est pas accompagnée de déperditions, d'imprécisions, et de lacunes, en raison même de la présence des systèmes impliqués dans cette relation.

Le figement, en tant que mécanisme cristallisant l'idiomaticité d'une langue, nous servirait d'exemple pour démontrer combien la traduction des SF est problématique. Pour ce faire, nous essayerons de montrer comment la linguistique est au 
cour de la traduction, comment le figement serait le phénomène le plus difficile à traduire et pour quelles solutions le traducteur pourrait opter.

\section{Le linguistique au cour de la traduction}

Il peut paraître un peu paradoxal de se demander si on peut se passer du linguistique quand on mène une réflexion sur la traduction. Malgré l'évidence de l'interdépendance entre la langue et la traduction, nous tenons à poser cette question parce que certains pensent que la langue n'est qu'un outil mis au service d'une pratique et que s'il y a un impact quelconque sur la qualité de la traduction, cela relèverait beaucoup plus de la compétence du traducteur que des limites imposées par les codes linguistiques impliqués dans la traduction. Sans remettre en question l'importance de la compétence du traducteur dans la qualité de la traduction, nous pensons que l'attention doit porter davantage sur les langues elles-mêmes et voir en quoi une meilleure connaissance des systèmes aiderait à mieux négocier les contenus à transférer d'une langue à une autre.

Dans toute opération de traduction, deux systèmes linguistiques au moins sont en place. Ne pas tenir compte du caractère structurel des faits linguistiques utilisés, c'est ignorer tous les rapports (relatifs au système) qu'entretiennent toutes les unités linguistiques de la langue, rapports qui ne sont pas nécessairement identiques d'une langue à l'autre. Il est actuellement démontré que l'essentiel du contenu à communiquer est assuré par le système inférentiel de chaque langue qui s'inscrit dans toutes les composantes. Même si le lexique se charge de la majeure partie des relations inférentielles, il n'en a pas l'exclusivité; certaines structures syntaxiques, des variations intonatives, des spécificités énonciatives et d'autres aspects concourent également à la constitution de la charpente inférentielle qui conditionne toute interprétation sémantique et, par conséquent, tout transfert des contenus signifiés dans une autre langue ${ }^{4}$. Si on part de ce constat, on se trouve dans l'obligation de tenir compte des structurations spécifiques de chaque langue afin de mieux respecter tout ce qui n'est pas dit mais pris de fait comme évident quand on s'exprime. Cet aspect linguistique est d'autant plus important qu'il ne relève pas de ce qui est directement et explicitement exprimé. Il arrive même que certains discours soient complètement structurés sur la base de choix inférentiels volontairement ambigus ou flous, où l'essentiel de l'information réside dans la manière dont le message est construit ${ }^{5}$. Nous nous limitons ici à ces questions; d'autres aspects sont également importants; nous mentionnons au passage tout ce qui relève des connotations, des niveaux de langue et de tout ce qui est marqué par rapport à la norme et à l'interaction verbale et des contraintes énonciatives. Notre propos ici n'est pas de détailler toutes ces questions. Rappelons simplement que le présupposé théorique chez tous ceux qui marginalisent le linguistique dans la traduction est que la langue serait un système où les relations sémiotiques entre signifiant et signifié seraient fondées sur l'univocité: à un signifiant un seul signifié et vice-versa. Tel n'est pas le cas dans les langues naturelles où les relations plurivoques sont de règle. Une telle caractéristique implique évidemment tout un jeu de transferts sémantiques au sein de la même langue déterminant la configuration générale de son lexique. C'est en tenant compte de tels phénomènes que les récentes études ont mis en exergue les notions de domaines structurants et domaines structurés. La détermination des différents types de domaines et la hiérarchie qui les 
gouverne aideraient énormément à comprendre les processus cognitifs qui président à la catégorisation du réel par la langue et qui privilégient certaines associations, pas d'autres.

Or, il est à remarquer que ces transferts ne sont pas que de nature strictement sémantique (le cas de la polysémie), ils impliquent également le figement qui, contrairement à la polysémie, demeure peu étudié en tant que mécanisme linguistique et rarement abordé sous l'angle de la traduction.

\section{Pourquoi le figement serait-il donc particulièrement difficile à traduire?}

Pour répondre à cette question, nous convoquons trois types de raisons: la première est linguistique, la deuxième est culturelle et la dernière est technologique.

S'agissant de la dimension linguistique, nous retenons les faits systémiques, pragmatiques et discursifs. S'il y a un processus qui implique la totalité du système linguistique, c'est bel et bien le figement. Puisque les formations syntagmatiques figées sont par définition polylexicales et bien formées, elles constituent de fait des échantillons comportant l'essentiel des informations relatives au fonctionnement du système. Elles portent en elles des informations précieuses sur la structure rythmique, syllabique et mélodique de la langue. Cela se vérifie aisément dans les assemblages du type formulaire et les déformations qu'ils connaissent sous l'impact de l'usage courant (redistribution accentuelle, compactage syllabique accompagnant la disparition de certains phonèmes, restructuration syllabique, liaisons obligatoires, phénomène d'assimilation et de dissimilation, etc.). Le lexique qui sature les positions de chaque formation syntagmatique véhicule, par sa position centrale, une bonne partie de la syntaxe (celle qui relève des positions et celle qui est révélée par les marques morphologiques), les contenus sémantiques lexicaux avec tout ce qu'ils comportent comme jeu de synonymie, d'antonymie et de paraphrase et comme contraintes d'emploi (comme celles des registres de langue), et les mécanismes d'opacification sémantique (notamment la métaphore, la métonymie et la synecdoque). Relèvent également du système tous les moyens d'expression explicite des relations logiques et de la hiérarchisation des prédicats, la régularité (ou l'irrégularité morphologique) de la langue, les catégories grammaticalisées par le système, etc. Autant d'éléments qui versent dans le spécifique, c'est-à-dire l'idiomatique (cf. S. Mejri 2004)!

Quand on évoque la pragmatique, on privilégie notamment les faits en rapport avec l'adéquation de l'énoncé et le reste des données de l'énonciation (contexte, protagonistes de l'échange verbal, stéréotypie, savoirs partagés, hiérarchie sociale, etc.). Deux types de séquences figées sont pragmatiquement très contraints: les énoncés formulaires et les proverbes. Les premières exigent une très bonne connaissance des nuances d'interprétation que l'emploi de chaque formule implique. Songeons aux degrés de familiarité et de respect, de neutralité et de manières affectées, dans les formules de salutation (salut!, bonjour, mes hommages!...) et tout ce que leur emploi connote comme type de relation entre les interlocuteurs! Quant aux proverbes, ils monopolisent «la sagesse», qui ne peut pas être de mise dans la bouche des enfants, par exemple. Leur emploi est souvent conditionné par des contraintes énonciatives comme le bon choix de la situation dans laquelle le proverbe doit être ancré, ou le rapport avec le contexte discursif dans lequel un énoncé proverbial peut jouer le rôle 
d'un énoncé récapitulatif (dans les titres ou en clausule), annonciateur (dans les titres ou les préambules) ou articulateur (à l'intérieur des textes).

Le figement est également important sur le plan strictement discursif. Les discours les moins neutres ne peuvent pas y échapper, et ce pour au moins trois raisons:

- l'importance quantitative des SF dans le lexique: leur nombre dépasse de très loin celui des unités monolexicales. Pour s'en convaincre, il suffit de chercher dans les dictionnaires de langue les équivalents polylexicaux à certains mots courants (cf. Mejri 1997a pour mourir);

- la grande fréquence des unités grammaticales (les locutions conjonctives, prépositionnelles et adverbiales);

- la nécessité des reprises anaphoriques lexicales structurant le discours qui impose le recours presque systématique aux unités polylexicales; l'exemple de l'alternance des verbes monolexicaux et des constructions équivalentes à verbe support illustre bien ce phénomène (Lejmi 2003).

Le concours de ces trois facteurs a pour résultat que les SF couvrent le cinquième des textes étudiés (Fiala et al. 1978).

Pour ce qui est de la dimension culturelle, même si elle ne peut pas être appréhendée d'une manière autonome par rapport au linguistique, elle constitue une facette importante du figement. L'accès y est possible grâce à trois outils: la stéréotypie, la catégorisation grammaticale et lexicale et le jeu inférentiel. Pour la stéréotypie, nous renvoyons aux travaux de Amossy (1997), Schapira (1999) et Saïd (2001). Rappelons simplement que si la langue joue un rôle identitaire sans conteste, c'est parce qu'elle véhicule des croyances partagées qui sont le fruit de manières spécifiques dans la catégorisation par le langage et qui ont pour conséquence des inférences propres. La catégorisation trouve parfaitement son illustration dans le grammatical, c'est-à-dire toutes les catégories syntaxiques fixées à l'aide d'outils spécifiques (cf. par exemple les travaux sur l'épicène, notamment celui de M. Ghariani Baccouche 2001). Il en est de même pour la catégorisation lexicale. Le système affixal peut nous fournir d'excellents exemples: pourquoi privilégier tel type d'expression temporelle, spatiale ou intensive et pas d'autres? Pourquoi imposer tel type de quantification (bouchée, poignée, brassé, cuillerée, etc.), d'espace (oliveraie, roseraie, rizière, etc.), et de temps (en arabe: $\underline{\text { douha }}$ : "en milieu de matinée», zawa:l: "début d'après-midi», etc.), etc. Derrière toutes les configurations que revêtent le réel et l'expérience du monde dans la langue réside l'arbitraire des expériences existentielles à l'origine de toute construction sémiotique qu'est la dénomination. De telles configurations décident de la charpente inférentielle du lexique (cf. R. Martin 1976, 1992 et 2001) qui conditionne en grande partie l'échange verbal.

C'est par le biais de ce dernier point que nous pourrions convoquer la dimension technologique. Deux écueils de taille se dressent devant le traitement automatique des langues: la polysémie et le figement; la polysémie parce que la machine doit sélectionner pour chaque emploi la signification adéquate et le figement parce qu'elle doit être en mesure de reconnaître les unités polylexicales et leur attribuer la signification globale correspondante. De la bonne sélection de la signification adéquate (le cas de la polysémie) et de la séquence syntagmatique (le cas du figement) dépend l'ensemble des inférences que l'analyseur sémantique doit être capable de construire. R. Martin explique en détail ce qui se passe dans le cas de la polysémie (2001), tout reste à faire pour le figement. 
Il apparaît clairement que le figement est un processus qui s'inscrit beaucoup plus que les autres mécanismes de la langue dans l'idiomaticité et dans ce qu'il y a de plus spécifique. Si la polysémie peut être considérée comme un outil équivalent, elle ne peut en aucune façon être aussi importante parce que le champ d'action du figement dépasse le sémantique pour l'englober et l'associer au lexical et au grammatical. La traduction, vue sous cet angle, devient une opération très difficile: plus on plonge dans le spécifique, moins la traduction serait aisée.

\section{Quelles solutions pour le traducteur?}

Il ne s'agit pas évidemment de fournir des solutions inédites. Les traducteurs ont mis à l'épreuve dans leurs pratiques toutes les solutions. Notre objectif est de nature beaucoup plus méthodologique. Notre intention est d'orienter la traduction du côté d'attitudes beaucoup plus modestes.

Si l'on fait abstraction des correspondances, qu'elles soient mono- ou polylexicales, le passage d'une langue à une autre se fait, surtout dans le cas du figement, avec des déperditions importantes contre lesquelles les solutions demeurent peu évidentes. Au lieu de chercher à contourner les difficultés par des artifices divers, il serait théoriquement plus pertinent d'avancer des propositions qui soient de nature à gérer au mieux le déficit en question et qui tiennent compte des modes de structuration lexicale dans chaque système linguistique. Nous fournissons dans ce qui suit trois types de solutions approchées qui ne sont certainement pas «idéales» mais qui présentent l'avantage d'être fructueuses parce qu'elles attirent l'attention sur les vrais problèmes linguistiques de la traduction, et ouvrent par la même occasion des perspectives devant la recherche dans ce domaine, où l'idéal de la traduction parfaite bloque toute tentative d'exploration linguistique ${ }^{6}$.

Pour ne pas perdre une grande partie du sémantisme de la SF, tout un travail doit se faire sur les équivalences possibles entre les unités lexicales, qu'elles soient polylexicales ou pas, des langues impliquées dans la traduction. Un tel travail, pour évident qu'il soit, s'il est fait systématiquement, fournira des détails précieux sur les possibilités d'adéquation entre les systèmes lexicaux. Rappelons à ce propos que les études sur la synonymie ont longuement souffert du même préjugé: à quoi bon étudier les équivalences si elles sont données comme telles par le système? Ce n'est qu'après avoir pris conscience que l'étude de la synonymie constitue une source inépuisable du nuancement du sens et qu'analyser les synonymes revient à en dégager les différences, que les travaux sur la synonymie ont commencé à avoir droit de cité en linguistique ${ }^{7}$. En traduction, le fait de dégager tout le jeu d'équivalences possibles entre les langues fournirait sûrement un outil très efficace pour réduire au maximum les déperditions pour des raisons strictement linguistiques.

Si les investigations n'aboutissent pas du côté de ce qui est conceptualisé lexicalement, il faut chercher à combler le déficit, soit au moyen d'autres outils que la langue offre, soit en ayant recours à la conceptualisation faite par et dans le discours. Pour le premier cas, on peut fournir l'exemple de certaines catégories générales exprimables par différents moyens. Tel est le cas de l'intensité (adverbe, répétition du même mot, morphème affixal), de la modalité (adverbes de point de vue, unités lexicales marquées, morphème du conditionnel, constructions syntaxiques, constructions corrélées), de la négation (syntaxe, morphologie, lexique, sémantisme des 
mots), etc. Dans le second cas, il faut se fier aux combinaisons discursives libres qui tentent de conceptualiser l'instantané à partir de ce qui est fixé dans la langue. C'est par ce biais que nous rejoignons la dernière solution, la paraphrase.

Il s'agit là d'une solution incontournable en traduction. Appréhendée d'habitude dans l'intralingual (cf. les travaux de Fuchs, notamment) comme outil de reformulation, elle peut être d'une très grande efficacité en traduction. La supériorité de la paraphrase réside dans la liberté de dosage sémantique laissée au locuteur, une liberté qui va de l'équivalence sémantique presque parfaite jusqu'à l'antonymie totale. Il n’y a pas de meilleure solution devant la gestion du déficit.

Nous pouvons dire en guise de conclusion que ces repères méthodologiques n'ont pour ambition que de soulever des problèmes très anciens, mais vus sous la double perspective: celle du figement, considéré comme un aboutissement ultime de l'idiomaticité, et celle du déficit, présenté comme une posture fructueuse en matière de recherche linguistique en rapport avec la traduction. Ils peuvent faire l'objet de développements plus substantiels et être illustrés par des traductions disponibles. Leurs implications, surtout dans la conception des programmes de formation de traducteurs et dans l'évaluation de la qualité des traductions, nous paraissent des plus évidentes.

\section{NOTES}

1. Cf. par exemple l'absence d'article devant le nom dans chercher noise, la syntaxe de sans coup férir et le mot fur dans au fur et à mesure. G. Gross parle dans ce cas de «blocs erratiques» (1996).

2. Cf. Hjelmslev pour l'opposition expression/contenu et forme/substance (1968).

3. Pour plus de détails sur la structuration du système linguistique par la polysémie et la polylexicalité, cf. Syntaxe et sémantique, no 5, Presses universitaires de Caen, 2003.

4. Cf. à propos de l'inférence les travaux de Robert Martin, notamment 1976, 1992 et 2001.

5. Cf. Robert Martin (1992) pour la notion de flou et (1976) pour les deux formes d'inférence, l'implication et la présupposition.

6. Avec le structuralisme, le recours à la traduction comme outil d'investigation linguistique était systématiquement banni. Nous assistons ces derniers temps à la réhabilitation de la traduction dans la méthodologie du linguiste, notamment dans le traitement automatique, la typologie des langues et les études contrastives.

7. Cf. par exemple l'importance de la synonymie dans la structuration du lexique, horizontalement avec les variantes régionales ou de niveau de langue et verticalement avec les emboîtements hyper-/ hyponymiques, et dans celle du discours avec l'anaphore lexicale.

\section{RÉFÉRENCES}

Amossy, R. (1997): Stéréotypes et clichés, Paris, Nathan Université.

Durieux, Ch. (2000): «Linguistique, traductologie, traductique. Une évolution singulière», $L a$ traduction: diversité linguistique et pratiques courantes, $2^{\text {ème }}$ volume des Actes du colloque Traduction humaine, traduction automatique, interprétation, Tunis, Publication du CERES.

Fiala, P. et al. (1978): «Des mots aux syntagmes. Figement et variation dans la Résolution générale du congrès de la CGT de 1978», Mots 14, pp. 47-88.

Fuchs, C. (1994): Paraphrase et énonciation, Paris, Ophrys.

Ghariani Baccouche, M. (2001): "L'autre, cet(te) épicène», in Clas, A., Awaiss, H. et J. Hardane, L'éloge de la différence: la voix de l'autre, Actualité scientifique, AUPELFUREF.

Gross, G. (1996): Les expressions figées en français, Paris, Ophrys.

Hjemslev, L. (1968): Prolégomènes à une théorie du langage, Paris, Minuit. 
Lederer, M. (1994): La traduction aujourd'hui, Paris, Hachette.

MARTIN, R. (1976): Inférence, antonymie et paraphrase, Paris, Klincksieck.

Martin R. (1992): Pour une logique du sens, Paris, PUF.

Martin R. (2001): Sémantique et automate, Paris, PUF.

Mejri, S. (1994): «Séquences figées et expressions de l'intensité», Cahiers de lexicologie 65-2, pp. 111-122.

MejRI, S. (1997a): Le figement lexical: descriptions linguistiques et structuration sémantique, Publications de la Faculté des lettres de la Manouba, volume X.

MejRI, S. (1997b) : «Défigement et jeux de mots», Études linguistiques 3, pp. 75-92.

Mejri, S., Gross, G., Clas, A. et T. Baccouche (1998a): Le figement lexical, Actes de la $1^{\text {re }}$ Rencontre Linguistique Méditerranéenne, Tunis, CERES.

MejRI, S. (1998b): «Les séquences figées: saillance linguistique et continuité référentielle», Scolia 11, pp. 169-179.

MejRI, S. (1998c): «La mémoire des séquences figées: une troisième articulation? ou la réhabilitation du culturel dans le linguistique», in Actes des Ve Journées scientifiques du réseau LTT de l'AUPELF-UREF, La mémoire des mots, pp. 3-11.

MejRI, S. (1999): «Polylexicalité et unité lexicale», LINX, 40, Nanterre-Paris X.

MejRI, S. (2000a): "Traduction, poésie, figement et jeux de mots», META 45-3, pp. 412-423.

MejRI, S. (2000b): «L'écriture littéraire bilingue: traduction ou réécriture? Le cas de Salah Guermadi», META, 45-3, pp. 450-457.

MejRI, S. (2000c) : «Figement et dénomination», META 45-4, pp. 609-621.

MejRI, S. (2000d): «Syntaxe et figement», Mélanges offerts à Gaston Gross, Bulag, numéro hors série, Presses universitaires de Franche-Comté, p. 333-342.

Mejri, S. et T. BAccouche (2000e): La traduction dans le monde arabe, numéro spécial, META 45-3.

MejRi, S. (2001a): «Traduire les jeux de mots: repères méthodologiques», Mélanges offerts à Jean-Marie Van der Meerschen, Bruxelles, Les éditions du Hazard.

MejRI, S. (2001b): «L'altérité dénominative», Revue tunisienne des sciences sociales 120, Centre d'Études et de Recherches Économiques et Sociales, Tunis.

MejRi, S. (2003a): Le figement lexical, numéro spécial, Cahiers de lexicologie 82-1.

MejRI, S. (2003b): Syntaxe et sémantique, Polysémie et polylexicalité 5, Université de Caen.

MejRI, S. (2003c): «La stéréotypie du corps dans la phraséologie. Approche contrastive», in Burger, H., Häcki Buhofer, A. et G. Gréciano, Phraseologie und Parömiologie, Band 14, Flut von Texten-vielfalt der Kulturen, Essen, Schneider Verlag Hohengehren GmbH, pp. 203-217.

MejRI, S. (2003d): «Le figement lexical», Cahiers de lexicologie 82-1, numéro spécial sur le figement lexical.

MejRI, S. (2003e) : «Polysémie et polylexicalité», Syntaxe et sémantique 5, Caen, Presses universitaires de Caen.

MejRi, S., Baccouche, T. Clas, A. et G. Gross (2003f): «La traduction linguistique: problème terminologique ou construction conceptuelle?», Traduire la langue, traduire la culture, $4^{\mathrm{e}}$ volume des Actes du colloque Traduction humaine, traduction automatique, interprétation, Paris, Maisonneuve et Larose, pp. 177-191.

Mejri, S., Baccouche, T. Clas, A. et G. Gross (2000a): La traduction: théories et pratiques, $1^{\text {er }}$ volume des Actes du colloque Traduction humaine, traduction automatique, interprétation, Tunis, Publication de l'ENS.

Mejri, S., Baccouche, T. Clas, A. et G. Gross (2000b): La traduction: diversité linguistique et pratiques courantes, $2^{\mathrm{e}}$ volume des Actes du colloque Traduction humaine, traduction automatique, interprétation, Tunis, Publication du CERES.

Mejri, S., Baccouche, T. Clas, A. et G. Gross (2001): La traduction, entre équivalence et correspondance, $3^{\mathrm{e}}$ volume des Actes du colloque Traduction humaine, traduction automatique, interprétation, Tunis, Publication de l'Institut supérieur des langues. 
252 MetA, LIII, 2, 2008

Mejri, S., Baccouche, T. Clas, A. et G. Gross (2003): Traduire la langue, traduire la culture, $4^{\mathrm{e}}$ volume des Actes du colloque Traduction humaine, traduction automatique, interprétation, Paris, Maisonneuve et Larose.

SAID, M. (2001): «La stéréotypie: un fait culturel ou linguistique?», La traduction, entre équivalence et correspondance, $3^{\mathrm{e}}$ volume des Actes du colloque Traduction humaine, traduction automatique, interprétation, Tunis, Publication de l'Institut supérieur des langues.

Schapira, Ch. (1999): Le stéréotypes en français, Paris, Ophrys. 